\title{
De novo genome sequencing, assembly, and analysis of three Russian Prunus persica varieties
}

Gladysheva-Azgari M.V. ${ }^{1 *}$, Slobodova N.V. ${ }^{1}$, Boulygina E.S. ${ }^{1}$, Tsygankova S.V. ${ }^{1}$, Sharko F.S. ${ }^{1}$, Mitrofanova I.V. ${ }^{2}$

${ }^{1}$ National Research Center "Kurchatov institute", Moscow, Russia

${ }^{2}$ Nikitsky Botanical Garden - National Scientific Center of the Russian Academy of Sciences (NBG-NSC RAS), Yalta, Russia

*email: marglader@gmail.com

Common peach (Prunus persica) is an important fruit crop for domestic agriculture, new varieties of which are actively developed by breeders of the Nikitsky Botanical Garden (NBG-NSC RAS). Currently, the main task of the breeders is the creation of fruit crops varieties resistant to abiotic and biotic environmental factors. Genome decoding is an integral part of plant genetic research. The peach genome ('Lovell' variety) has recently been sequenced and annotated in very high quality. There are sequences of hundreds of peach varieties posted in the global resource, which are available to the international scientific community. However, these resources lack information on the sequencing of varieties of domestic selection and varieties from the collection of the NBG-NSC RAS. Therefore, this study is relevant both for further selection, as well as for the subsequent widespread introduction of new varieties into the horticulture of Russia. Three P. persica varieties ('Sovietskiy', 'Dostojnij', 'Babygold-7') were selected for genome sequencing, varieties 'Sovietskiy' and 'Dostojnij' were created in Russia. Each selected variety represents its own ecological-geographic group for the further possibility of finding genes responsible for important agricultural traits for different growing areas. High molecular weight DNA were isolated from the collected material (mature leaves of three $P$. persica varieties). Corresponding DNA libraries were sequenced using NovaSeq 6000 Illumina and Oxford Nanopore GridON. The obtained data were used for hybrid assemblies of three $P$. persica varieties and subsequent analysis of the resulting sequences for various mutations. Further, these genomes can be used as reference genomes for the genotyping of the entire peach collection, including the identification of target genes/loci responsible for resistance and adaptive properties in southern Russia. 